\section{Atrophe Aknenarben: Schrittweise zum Erfolg}

Im Gegensatz zu wulstigen Narben und Keloiden sind Aknenarben meist atroph. Eine einzige Laserbehandlung alleine genügt da oft nicht.

A trophe Narben sind bei Patienten, gerade auch bei Männern, ein großes Thema, weiß Dr. Tanja Fischer, Potsdam, aus ihrer eigenen Praxis. Bei der Therapie tiefer Aknenarben wendet sie vor dem fraktionierten $\mathrm{CO}_{2}-$ Laser einen nonablativen Erbium-GlassLaser (Fraxel Restore) bei hoher Energie ein bis zwei Mal im Abstand von 4 Wochen an. Um die Lichtbrechung an sehr kantigen, stufigen Narbenrändern zu verringern, trägt Fischer teilweise vor der

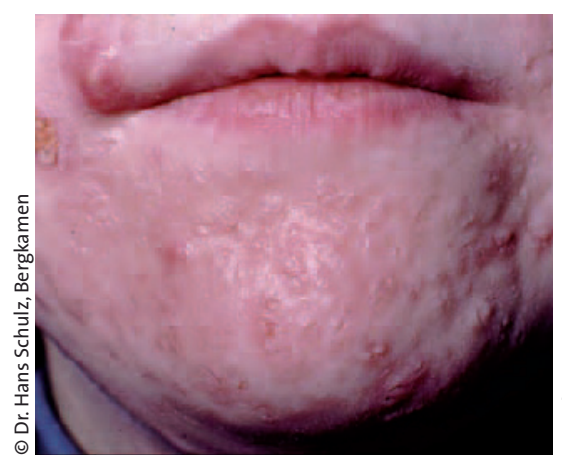

eigentlichen Behandlung des Gesamtbilds zunächst die Narbenkanten mit dem Erbium YAG-Laser ab und erzielt so bereits eine optische Verbesserung. Teilweise macht sie dann auch schon eine Subzision und eine leichte Hyaluronsäureunterfütterung. Bei der Subzision löst sie mit einer scharfen Nadel den Narbengrund mechanisch, ehe sie die Hyaluronsäure injiziert. Zwei Wochen später folgt dann die Behandlung mit dem fraktionierten Erbium Glass- und $\mathrm{CO}_{2}$-Laser.

Kurs „Probleme mit Narben - aktuelle Therapiemöglichkeiten“. 27.7.2010

Teils ist der gestaffelte Einsatz verschiedener Lasertypen hier sinnvoller.

\section{Narbentherapie: Was bringt Silikon wirklich?}

Silikongele und -folien finden verbreitet Einsatz in der Narbenbehandlung. Über den Wirkmechanismus wird spekuliert, die Evidenz für die Wirksamkeit ist beschränkt.

$\mathrm{N}$ ach internationalen Empfehlungen kommen Silikongele und Silikongelfolien als primäre Therapieoption bei hypertrophen Narben und Keloiden zum Einsatz (Grafik). Silikongelfolien sollen mindestens 12, besser 24 Stunden pro Tag über mindestens 2 Monate getragen werden. Erst dann kann das Ansprechen beurteilt werden. Die Kombination mit Kompressionsmethoden ist möglich. Die Ansprechraten in der Literatur schwanken erheblich, wie Prof. Dr. Sigrid Karrer, Regensburg, kritisch anmerkte: Es existieren Angaben zwischen 60 und $100 \%$. Silikongele sind leichter zu applizieren als die Folien, sollen zweimal am Tag dünn aufgetragen werden und gelten als indiziert bei hypertrophen Narben und Keloiden, wo sie aber nicht viel bringen, meint Karrer. Eher indiziert sind sie zur Prophylaxe und Rezidivprophylaxe bei Neigung zu hypertrophen Narben und Keloiden. Über den Wirkmechanismus weiß man erstaunlich wenig: Es wird ein okklusiver Effekt vermutet, die entstehende feuchte Kammer soll dazu führen, dass
Wachstumsfaktoren vermehrt ausgeschüttet werden.

\section{Belege nur für Prophylaxe mit Folien}

Eine Cochrane-Metaanalyse von 15 kontrollierten Studien fand für die Wirksamkeit der prophylaktischen Anwendung der Silikongelfolien eine gewisse Evidenz: Nach Operationen schienen hypertrophe Narben so seltener aufzutreten [O'Brien L, Pandit A. Cochrane Database Syst Rev. 2006CD003826].
Beim therapeutischen Einsatz der Folien scheinen die Narbenhöhe und das Erythem reduziert werden zu können, die Autoren der Metaanalyse betonten aber, dass die Qualität der Studien zu gering ist, um den Effekt hier wirklich beurteilen zu können. Für die Gele gibt es keine solchen Analysen. Für die Silikongelfolien und Gele spricht laut Karrer die einfache Anwendung. Sie sind nicht invasiv und damit auch für Kinder geeignet, allerdings muss der Patient eine gewisse Compliance mitbringen, wie Karrer betonte, weil Gele wie Folien konsequent über mehrere Monate angewendet werden müssen. Dabei ist die Wirksamkeit letztlich begrenzt.

Kurs „Probleme mit Narben - aktuelle Therapiemöglichkeiten“, 27.7.2010

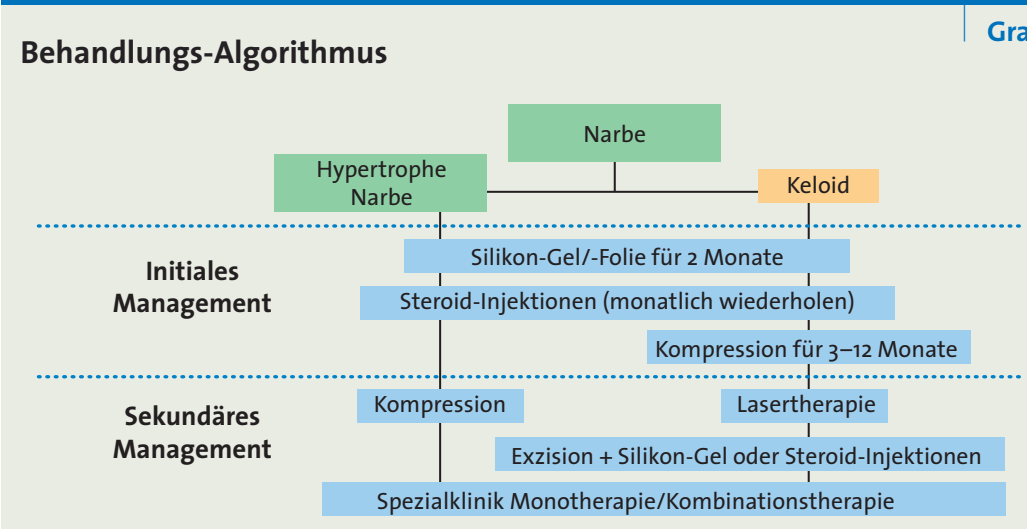

